\title{
The Regulation of ApoB Metabolism by Insulin
}

\author{
Mary E. Haas ${ }^{1}$, Alan D. Attie ${ }^{2}$, and Sudha B. Biddinger ${ }^{1}$ \\ ${ }^{1}$ Division of Endocrinology, Boston Children's Hospital, Harvard Medical School, Boston \\ Massachusetts, USA, 02115 \\ ${ }^{2}$ Department of Biochemistry, University of Wisconsin-Madison, Madison, Wisconsin, USA, 53706
}

\begin{abstract}
The leading cause of death in diabetic patients is cardiovascular disease. Apolipoprotein B (ApoB)-containing lipoprotein particles, which are secreted and cleared by the liver, are essential for the development of atherosclerosis. Insulin plays a key role in the regulation of ApoB. Insulin decreases ApoB secretion by promoting ApoB degradation in the hepatocyte. In parallel, insulin promotes clearance of circulating $A p o B$ particles in the liver via the low density lipoprotein receptor (LDLR), LDLR-related protein 1 (LRP1), and heparan sulfate proteoglycans (HSPGs). Consequently, the insulin resistant state of Type 2 diabetes (T2D) is associated with increased secretion and decreased clearance of ApoB. Here, we review the mechanisms by which insulin controls the secretion and uptake of ApoB in normal and diabetic livers.
\end{abstract}

\section{Keywords}

apolipoprotein B; insulin; diabetes; very low density lipoprotein (VLDL); cardiovascular disease; selective insulin resistance

\section{Cardiovascular Disease in Diabetes}

Most individuals with diabetes ultimately die of cardiovascular disease (CVD). Hyperglycemia is a central feature of the diabetic state, and it has been suggested that hyperglycemia promotes cardiovascular disease by increasing inflammation, oxidative stress and the formation of advanced glycosylation end products (for review, see [1]). However, the normalization of serum glucose levels does not consistently reduce the risk of CVD in diabetic patients [2,3]. A more important factor may be derangements in lipoprotein metabolism. Although diabetic patients are not usually hypercholesterolemic, they do show abnormalities in lipoprotein kinetics and composition. Moreover, statin drugs, which reduce serum cholesterol, effectively reduce CVD risk in diabetic patients [4]. Here, we will review the effects of insulin on the hepatic metabolism of apolipoprotein B (ApoB)-containing lipoprotein particles, as these are essential for the development of atherosclerosis.

(C) 2013 Elsevier Ltd. All rights reserved.

Corresponding Author: Biddinger, S.B. (sudha.biddinger@childrens.harvard.edu).

Publisher's Disclaimer: This is a PDF file of an unedited manuscript that has been accepted for publication. As a service to our customers we are providing this early version of the manuscript. The manuscript will undergo copyediting, typesetting, and review of the resulting proof before it is published in its final citable form. Please note that during the production process errors may be discovered which could affect the content, and all legal disclaimers that apply to the journal pertain. 


\section{Overview of Lipoprotein Metabolism}

The lipoprotein system allows the intercellular transport of cholesterol and triglycerides, which serve distinct roles in the body. Triglycerides are an important source of energy. Cholesterol, on the other hand, is a structural element of membranes as well as a precursor for steroids and bile acids. In contrast to triglycerides, most mammalian tissues are not capable of catabolizing cholesterol. They must therefore return excess cholesterol to the liver, which can secrete cholesterol into the bile, either directly or after conversion to bile acids [5].

Lipoproteins can be divided into classes that vary in their apolipoproteins, lipid composition and, therefore, function. Apolipoprotein B is a structural protein that is an integral component of chylomicrons, as well as very low density lipoprotein (VLDL), intermediate density lipoprotein (IDL) and low density lipoprotein (LDL) particles. Chylomicrons are secreted by the intestine and enable the transport of dietary triglycerides to other tissues after feeding. They contain a truncated form of ApoB, referred to as ApoB 48 as it is $48 \%$ of the full-length protein [6]. Chylomicrons are lipolyzed within the vasculature, releasing their fatty acids to peripheral cells, particularly the adipose tissue, for storage. When most of the triglycerides have been removed, the chylomicron remnant is cleared by the liver [5].

VLDL, which is secreted by the liver, also transports triglycerides. In man, VLDL contains only ApoB100, the full length protein, whereas either ApoB100 or ApoB48 is present in the VLDL of mice and certain other rodents [6]. As the VLDL particle delivers triglycerides to other tissues, it is successively lipolyzed into an IDL and then LDL particle. LDLcholesterol can be taken up by cells that require exogenous cholesterol. However, most LDL is cleared by the liver [5].

The other major class of lipoproteins is high density lipoprotein (HDL). HDL does not contain ApoB, and high HDL levels are correlated with decreased risk of atherosclerosis. HDL can remove excess cholesterol from peripheral tissues, such as endothelial cells and macrophages, and return it to the liver for excretion from the body, a process known as reverse cholesterol transport [7].

Insulin plays a central role in coordinating lipoprotein metabolism. In the fed state, triglycerides and other nutrients are available from the gut. In this case, high insulin levels act on the adipocyte to promote triglyceride uptake and inhibit free fatty acid release. This promotes the storage of triglycerides for later use. In fasting, this process is reversed: insulin levels fall, and free fatty acids are released from the adipocyte and delivered to the liver. The liver re-esterifies the fatty acids to triglycerides and secretes them as VLDL [7]. Moreover, the ability of the adipose to take up triglycerides is impaired in the absence of insulin, and the triglycerides are therefore preferentially utilized by other tissues, such as skeletal muscle and liver $[7,8]$.

\section{Regulation of VLDL production}

Within the hepatocyte, insulin exerts its effects via a complex signaling cascade [9]. Some of the major branches of this cascade are shown in Figure 1. Upon binding insulin, the insulin receptor recruits the insulin receptor substrate (IRS) proteins, leading to activation of phosphatidylinositide 3-kinase (PI3K), which mediates many of insulin's metabolic effects.

PI3K activates Akt, which inhibits the transcription factor FoxO1 by phosphorylating it. Phosphorylated FoxO1 is excluded from the nucleus, and therefore unable to activate transcription of its targets, including the gluconeogenic genes. Akt also stimulates glycogen synthesis by inhibiting glycogen synthase kinase 3 (GSK3). Furthermore, Akt activates 
lipogenesis, in part via the mammalian target of rapamycin complex 1 (mTORC1) signaling complex, which is necessary for the induction of the lipogenic transcription factor Sterol Regulatory Element Binding Protein (SREBP)-1c [10]. Consequently, in the hepatocyte, insulin suppresses glucose production and stimulates the production of glycogen and triglycerides. It also acutely inhibits the secretion of ApoB [11].

The synthesis and secretion of ApoB is complex. Surprisingly, most ApoB protein is degraded prior to secretion, and the amount of ApoB secreted is largely determined by the proportion of newly synthesized polypeptide that escapes degradation [6]. There are multiple mechanisms by which insulin regulates ApoB (Figure 2). Though insulin does not alter $A p o B$ mRNA levels [12], it inhibits ApoB translation by promoting the trafficking of $A p o B$ mRNA into P-bodies, aggregates of translationally repressed mRNAs $[13,14]$. The effects of insulin on ApoB translation require signaling through PI3K and mTORC1, and may involve the association of RNA binding proteins with the $A p o B$ mRNA 5'UTR [15].

As ApoB is translated, it is lipidated by microsomal triglyceride transfer protein (MTP). MTP adds triglycerides to the nascent ApoB during its co-translational translocation into the lumen of the endoplasmic reticulum [16]. This produces a dense, lipid-poor, pre-VLDL particle. The expression of MTP is driven by FoxO1, and thus inhibited by insulin [17]. As ApoB must be lipidated by MTP in order to escape proteosomal degradation [16], insulin may promote ApoB degradation by decreasing expression of MTP. Consistent with this, insulin resistant rodents show increased amounts of nuclear FoxO1, MTP mRNA, and MTP protein, as well as increased ApoB secretion [17,18]. However, acute insulin treatment in rodents does not decrease either MTPmRNA or MTP activity [19]; this may due to the long half-life of MTPmRNA, approximately 4.4 days [20]. ApoB may also be regulated by ER60, an endoplasmic reticulum resident chaperone and protease that binds and degrades ApoB when overexpressed [21]; interestingly, ER60 is decreased and ApoB secretion is increased in the insulin resistant fructose-fed hamster [22].

A second lipidation step, in which the bulk of lipids are added to ApoB, occurs independently of MTP $[11,23]$. This maturation step can be inhibited by insulin/PI3K signaling [24]. Although the exact mechanisms are unknown, one potential mediator is ApoCIII, an apolipoprotein secreted by the liver and to a lesser extent by the intestine. Individuals with a null allele of ApoCIII manifest a $45 \%$ reduction in serum triglycerides and a $27 \%$ decrease in coronary heart disease risk [25]. ApoCIII is best known for its ability to inhibit lipoprotein lipase and hepatic lipase, and therefore lipolysis of triglycerides carried on chylomicrons and VLDL [26], but it has also been suggested to act intracellularly to promote ApoB secretion. Expression of ApoCIII in McA-RH7777 rat hepatoma cells, which lack endogenous ApoCIII, increases ApoB and triglyceride secretion by promoting the second step of ApoB lipidation [27]. However, ApoB secretion in vivo is not increased by ApoCIII expression and VLDL triglyceride secretion is increased only upon expression of very high levels of ApoCIII [28].

Transcription of $A p o C I I I$, like $M T P$, is induced by FoxO1, and therefore inhibited by insulin. ApoCIII transcription is also induced by the carbohydrate response element binding protein (ChREBP), which is activated by glucose [29], and peroxisome proliferatoractivated receptor gamma coactivator $1-\beta$ (PGC-1 $\beta$ ), which is activated by fatty acids [30]. In the diabetic state, the presence of insulin resistance, hyperglycemia and elevated free fatty acids would all be expected to drive ApoCIII transcription. Indeed, ApoCIII levels are increased in the serum of patients with T2D [31].

The triglycerides used for ApoB lipidation are derived from three sources: free fatty acids released from the adipocyte, hepatic uptake of chylomicron and VLDL remnants, and de 
novo lipogenesis. Interestingly, insulin has opposing effects on the different sources of hepatic triglycerides. Though insulin decreases the free fatty acid supply to the liver by suppressing adipose tissue lipolysis, it promotes the uptake of remnant particles (see below) and lipogenesis (for review see [11]).

The lipidated ApoB particle can also be degraded before being secreted, and this may be a more important site of insulin action, at least in vitro. Though the exact mechanisms are again unclear, insulin stimulation of ApoB degradation is blocked by brefeldin A, which inhibits intracellular transport between the endoplasmic reticulum and the Golgi [32], as well as by knockout of Atg5, a protein necessary for autophagy [33].

Insulin may also regulate the lipidated ApoB particle via sortilin [34, 35]. Sortilin is a member of the Vps10p-domain family of transmembrane receptors, which bind and traffic extracellular ligands to the lysosome; sortilin can also traffic Golgi-localized ligands to the lysosome [36]. Sortilin appears to target ApoB to the lysosome for degradation, as the ability of sortilin to inhibit ApoB secretion is blocked by mutations that interfere with sortilin trafficking to the lysosome and by disrupters of lysosome function [34]. Consistent with this, sortilin overexpression decreases VLDL secretion in most, though not all, studies $[34,37,38]$. Surprisingly, recent work has shown that sortilin is suppressed by mTORC1 [38], a downstream target of insulin. This suggests that insulin may actually promote ApoB secretion via sortilin, though overall it inhibits ApoB secretion via other pathways.

Finally, insulin can antagonize ApoB secretion by promoting the re-uptake of secreted VLDL back into the hepatocyte before it exits the space of Disse, located between the hepatocyte and endothelial cell. This re-uptake is mediated by the LDL receptor and potentially HSPGs [39,40]. If the VLDL particle escapes re-uptake, it can enter the circulation and deliver its triglyceride cargo to other tissues.

\section{Regulation of Lipoprotein Clearance}

ApoB particles are removed from the circulation primarily by the liver; this process is promoted by insulin and impaired in diabetes [41-43]. Here, we will examine the effects of insulin on the hepatic receptor systems that mediate ApoB clearance: the LDL receptor, LRP1, and HSPGs. However, it is important to note that other factors in the diabetic state could also contribute to delayed ApoB clearance. First, there is a defect in triglyceride lipolysis. Second, hyperglycemia and increased oxidative stress promote glycosylation and oxidization of lipoprotein particles, which could interfere with their uptake and clearance $[44,45]$. Finally, the overproduction of lipoproteins from the liver as well as the intestine (for review, see [46]) could indirectly impair lipoprotein clearance by competing for the clearance apparatus.

\section{LDL receptor}

The LDL receptor (LDLR), which binds ApoB100 and ApoE, is a key determinant of serum cholesterol levels and cardiovascular disease risk. Over thirty years ago, insulin was shown to promote LDL uptake in fibroblasts [47,48]. Subsequent studies in cultured hepatoma cells showed that insulin increases $L D L R$ mRNA [49]. The effects of insulin on LDLR mRNA appear to be mediated by the transcription factor SREBP-1c, which also mediates insulin's effects on lipogenesis. Thus, in the livers of rodents with decreased insulin levels due to fasting [50] and Type 1 diabetes (T1D) [51], Ldlr mRNA levels are decreased.

The effects of insulin on LDLR protein, however, are less clear. The livers of mice with liver-specific knockdown [52] or knockout [53] of the insulin receptor show reduced levels 
of Ldlr protein. On the other hand, Ldlr protein levels in the liver do not change with fasting or T1D [50,51], and reports of Ldlr protein levels in obesity/T2D are conflicting [52,54].

The discordance between LDLR mRNA and protein levels under certain circumstances could be due to proprotein convertase subtilisin/kexin type 9 (PCSK9). PCSK9 is a secreted protein that binds to the LDLR and promotes its degradation. Pcsk 9 knockout mice have increased levels of Ldlr protein and therefore increased LDL clearance [55]. Humans with gain of function mutations in PCSK9 show increased levels of LDL-cholesterol and increased risk of cardiovascular disease, whereas individuals with loss of function mutations in PCSK9 show decreased levels of LDL-cholesterol and reduced risk of cardiovascular disease [55]. Moreover, therapies to inhibit PCSK9 have recently been shown to be effective in reducing LDL-cholesterol levels [56].

Interestingly, insulin increases PCSK9 mRNA in vitro [50]. In vivo, fasting and T1D decrease insulin and $P \operatorname{csk} 9$ mRNA [50,51]. In these models, the reduced expression of Pcsk9 has been invoked to account for the fact that the abundance of Ldlr protein does not change even as Ldlr mRNA is decreased. Taken together, these data illustrate the fact that the effects of insulin and diabetes on LDLR are complex, as the ability of insulin to induce PCSK9 could reduce LDLR expression, even as insulin action on SREBP-1c and other pathways promotes LDLR expression.

\section{Low Density Lipoprotein Receptor-Related Protein 1 (LRP1)}

LRP1 is another member of the LDLR family. It contains a very large extracellular domain capable of binding more than thirty ligands, including lipoproteins, growth factors, and protease inhibitors [57]. Though LRP1 is unable to bind ApoB and therefore LDL, it is able to bind remnant particles via molecules such as ApoE [57]. However, the role of LRP1 in lipoprotein clearance remains unclear. In $L d l r$ deficient mice, liver-specific knockout of Lrp1 leads to impaired removal of chylomicron remnants and a two- to three-fold increase in plasma cholesterol and triglycerides [58]. In mice that are wildtype for $L d l r$, however, liverspecific knockout of $\operatorname{Lrp} 1$ has little effect on plasma lipoproteins [58]. Thus, LRP1 is not necessary for lipoprotein clearance in the presence of functional LDLR.

Insulin has been shown to promote the translocation of LRP1 from intracellular vesicles to the cell surface $[59,60]$. Thus, treatment with glucose, which induces insulin secretion, increases LRP1 protein in plasma membrane preparations [59]. It also increases uptake of the LRP1 ligands, a2-macroglobulin and chylomicron remnants. The ability of insulin to promote a2-macroglobulin uptake is blunted in $o b / o b$ mice, a model of T2D, suggesting that obesity and insulin resistance could cause impaired LRP1 translocation [59]. In the adipocyte, LRP1 exists in the same vesicles as the Glut4 transporter, the major mediator of insulin-sensitive glucose transport [61], suggesting that the same signaling mechanisms utilized by insulin to translocate Glut4 may be used to translocate LRP1.

Interestingly, LRP1 also mediates the clearance of activated Factor VIII, which promotes clotting: mice deficient in $\operatorname{Lrp} 1$ show increased levels of circulating Factor VIII and decreased Factor VIII clearance [62]. LRP1 also appears to increase HDL-cholesterol, as hepatic knockout of Lrp1 decreases HDL synthesis and lowers HDL levels [63]. Thus, defects in LRP1 could potentially account for multiple features of obesity/T2D: a procoagulant state, low HDL-cholesterol and, if there are concomitant defects in the LDLR, post-prandial lipemia.

\section{Heparan sulfate proteoglycans (HSPGs)}

HSPGs consist of a core polypeptide onto which numerous negatively charged sugar moieties, heparan sulfates, are attached [64]. The result is a large, highly negatively charged, 
flexible structure. In the liver, HSPGs are positioned to trap lipoproteins within the space of Disse, rapidly clearing them from the circulation; they may also promote the uptake of these particles into hepatocytes by internalizing them directly, or facilitating their interaction with other receptors $[64,65]$. The liver expresses multiple HSPGs, including perlecan, syndecans 1, 2, and 4, and glypicans 1 and 4 [66]. The role of HSPGs, particularly syndecan-1, in lipoprotein clearance has been suggested by the findings that liver-specific knockout of either syndecan-1 or GlcNAc N-deacetylase/ $N$-sulfotransferase-1 (Ndst1), an enzyme that participates in the assembly of heparan sulfate moieties, can reduce clearance of remnant lipoproteins [66,67]. The reduction in remnant clearance occurs even in mice wildtype for Ldlr, indicating that deficiencies in the HSPGs, unlike Lrp1, cannot be compensated for by Ldlr.

Over 30 years ago, insulin deficiency was found to produce defects in the sulfation of proteoglycans [68], though this has not been found in all studies [69]. Moreover, perlecan protein and Ndst 1 mRNA are decreased in insulin-deficient rodents [70,71]. Additionally, $d b / d b$ mice, a model of T2D, show increased expression of heparan sulfate glucosamine 6O-endosulfatase 2 (Sulf2), an enzyme that promotes HSPG degradation [72]. Knockdown of Sulf 2 in $d b / d b$ mice increased the clearance of chylomicron remnants and lowered plasma triglyceride levels [73].

\section{Other players}

The lipolysis-stimulated lipoprotein receptor (LSR), which is expressed primarily in hepatocytes, promotes the uptake of ApoB and ApoE containing lipoproteins in the presence of high concentrations of fatty acids [74]. Interestingly, Lsr is decreased in mouse models of T1D [75] and T2D [76]. Knockdown of Lsr using adenoviruses encoding shRNA against Lsr increased serum triglycerides, whereas adenoviral reconstitution of Lsr in $o b / o b$ mice lowered serum triglyceride and cholesterol levels [76]. Second, ApoCIII interferes with the binding of ApoB particles to the LDL receptor, preventing their removal from the circulation [77]; this is in addition to its effects on lipolysis and ApoB secretion. Finally, sortilin not only inhibits ApoB secretion but also promotes the clearance of ApoBcontaining lipoproteins, as overexpression of sortilin in the liver increases, and knockout of sortilin decreases, the fractional catabolic rate of LDL [34]. Consistent with this, genomewide association studies in humans have shown that polymorphisms predicted to increase sortilin expression are strongly associated with lower LDL-cholesterol levels [78].

\section{The Diabetic Dyslipidemia}

Given the central role of insulin in the control of ApoB lipoprotein metabolism, it is not surprising that derangements in insulin signaling produce dyslipidemia. Indeed, liverspecific knockout of the insulin receptor increases secretion of ApoB, decreases LDL clearance, and markedly increases susceptibility to atherosclerosis in mice [53].

T2D is the most common form of diabetes and is associated with obesity. It is primarily due to insulin resistance - i.e., the failure of insulin to maintain glucose homeostasis - resulting in hyperglycemia and a compensatory hyperinsulinemia. For example, the failure of insulin to suppress FoxO1 promotes gluconeogenic gene expression and hyperglycemia. Interestingly, however, not all components of the insulin signaling cascade become impaired in T2D, a phenomenon known as "selective insulin resistance". For example, mTORC1 remains active in T2D, presumably because it either remains sensitive to insulin and is driven by the hyperinsulinemia present [79], or is activated directly by excess nutrients [80].

In normal physiology, insulin suppresses FoxO1 in the liver, decreasing MTP and ApoCIII expression, and therefore, ApoB secretion. Insulin also suppresses lipolysis in the adipose 
tissue, decreasing fatty acid flux to the liver. Although insulin also stimulates lipogenesis and suppresses sortilin, the net effect of insulin is to suppress ApoB secretion. With the development of T2D, however, FoxO1 becomes dis-inhibited, leading to increased expression of MTP and ApoCIII (Figure 3). At the same time, mTORC1 is activated, suppressing sortilin. Consistent with this, the ability of insulin to suppress ApoB secretion is lost in humans with T2D [81,82] and ApoB secretion is increased [81,83-86].

T2D also promotes the accumulation of hepatic triglycerides, which fuel the lipidation of ApoB. First, the ability of insulin to suppress lipolysis in the adipocyte is lost in T2D, leading to increased free fatty acid flux to the liver. Second, the ability of insulin to stimulate mTORC1, SREBP-1c and hepatic lipogenesis appears to remain intact [87]. The resulting excess of triglycerides promotes the formation of the VLDL1 subclass of particles, which are larger and more triglyceride rich than the VLDL2 subclass of particles $[11,88,89]$. Consistent with this, patients with T2D show an increase in the secretion of VLDL1, rather than VLDL2, particles $[83,90]$. Finally, T2D is associated with a decrease in triglyceride lipolysis [91] as well as ApoB clearance [42,43].

Hypertriglyceridemia is therefore common in T2D. Hypertriglyceridemia promotes the development of small, dense LDL, and decreased HDL via the enzyme cholesterol ester transfer protein (CETP). CETP, which is not present in mice and rats, catalyzes the transfer of triglycerides in VLDL for cholesterol esters in LDL and HDL, resulting in triglyceriderich LDL and HDL particles. Triglyceride-rich LDL particles can be lipolyzed to small, dense LDL, which are thought to be highly atherogenic because of their greater susceptibility to oxidation, reduced affinity for the LDL receptor, and increased uptake by arterial tissue [45]. Triglyceride-rich HDL particles are more susceptible to degradation, leading to lower HDL-cholesterol levels. Thus, increased VLDL1 secretion is central to the pathogenesis of all of the features of the dyslipidemia of T2D: hypertriglyceridemia, decreased HDL-cholesterol and increased small dense LDL-cholesterol. Note that hypercholesterolemia, though an established risk factor for cardiovascular disease, is not usually present in patients with T2D [92].

T1D, on the other hand, is due to autoimmune destruction of the beta-cells of the pancreas, leading to hypoinsulinemia and decreased insulin action on all pathways. Consequently, lipogenesis is decreased in rodent models of T1D [93]. Nonetheless, patients with untreated T1D have decreased lipoprotein clearance and hypertriglyceridemia [94,95]; those with poorly controlled T1D also have hypercholesterolemia, and increased VLDL-cholesterol and LDL-cholesterol $[96,97]$. Well controlled T1D patients, who are made insulin-replete by injections of exogenous insulin, however, generally show normal plasma triglycerides and cholesterol, normal or decreased LDL-cholesterol, and normal or increased HDL-cholesterol $[96,97]$, indicating that exogenous insulin is sufficient to restore lipoprotein homeostasis.

\section{Concluding Remarks and Future Perspectives}

In summary, insulin plays a central role in the secretion and clearance of ApoB lipoproteins, which play a key role in promoting atherosclerosis. Though diabetes is not usually associated with hypercholesterolemia, there are important changes in lipoprotein kinetics, including increased secretion and decreased clearance of ApoB particles. Consequently, the number of LDL particles, which is closely tied to atherosclerosis risk, is elevated [98]. Moreover, qualitative differences in LDL - i.e., the formation of small dense LDL, which are potentially intrinsically more atherogenic - are present. Thus, even though the development of cardiovascular disease is undoubtedly multi-factorial, defining and reversing the changes in ApoB metabolism that occur in diabetes could prove most effective in reducing mortality. 


\section{References}

1. Bornfeldt KE, Tabas I. Insulin resistance, hyperglycemia, and atherosclerosis. Cell metabolism. 2011; 14:575-85. [PubMed: 22055501]

2. Gerstein HC, et al. Effects of intensive glucose lowering in type 2 diabetes. The New England journal of medicine. 2008; 358:2545-59. [PubMed: 18539917]

3. Stocker R, Keaney JF. Role of oxidative modifications in atherosclerosis. Physiological reviews. 2004; 84:1381-478. [PubMed: 15383655]

4. Collins R, et al. MRC/BHF Heart Protection Study of cholesterol-lowering with simvastatin in 5963 people with diabetes: a randomised placebo-controlled trial. Lancet. 2003; 361:2005-16. [PubMed: 12814710]

5. Semenkovich, CF., et al. Disorders of Lipid Metabolism. In: Mehmed, S.; Williams, RH., editors. Williams Textbook of Endocrinology. 12. Elsevier/Saunders; 2011. p. 1633-1674.

6. Blasiole DA, et al. The physiological and molecular regulation of lipoprotein assembly and secretion. Molecular biosystems. 2007; 3:608-19. [PubMed: 17700861]

7. Bamba V, Rader DJ. Obesity and atherogenic dyslipidemia. Gastroenterology. 2007; 132:2181-90. [PubMed: 17498511]

8. Teusink B, et al. Contribution of fatty acids released from lipolysis of plasma triglycerides to total plasma fatty acid flux and tissue-specific fatty acid uptake. Diabetes. 2003; 52:614-20. [PubMed: 12606500]

9. Biddinger SB, Kahn CR. From mice to men: insights into the insulin resistance syndromes. Annual review of physiology. 2006; 68:123-58.

10. Li S, et al. Bifurcation of insulin signaling pathway in rat liver: mTORC1 required for stimulation of lipogenesis, but not inhibition of gluconeogenesis. Proceedings of the National Academy of Sciences of the United States of America. 2010; 107:3441-6. [PubMed: 20133650]

11. Choi SH, Ginsberg HN. Increased very low density lipoprotein (VLDL) secretion, hepatic steatosis, and insulin resistance. Trends in endocrinology and metabolism. 2011; 22:353-363. [PubMed: 21616678]

12. Dashti N, et al. Effects of oleate and insulin on the production rates and cellular mRNA concentrations of apolipoproteins in HepG2 cells. Journal of lipid research. 1989; 30:1365-73. [PubMed: 2689548]

13. Karimian Pour N, Adeli K. Insulin silences apolipoprotein B mRNA translation by inducing intracellular traffic into cytoplasmic RNA granules. Biochemistry. 2011; 50:6942-50. [PubMed: 21721546]

14. Parker R, Sheth U. P bodies and the control of mRNA translation and degradation. Molecular cell. 2007; 25:635-46. [PubMed: 17349952]

15. Sidiropoulos KG, et al. Insulin inhibition of apolipoprotein B mRNA translation is mediated via the PI-3 kinase/mTOR signaling cascade but does not involve internal ribosomal entry site (IRES) initiation. Archives of biochemistry and biophysics. 2007; 465:380-8. [PubMed: 17698027]

16. Fisher EA, Ginsberg HN. Complexity in the secretory pathway: the assembly and secretion of apolipoprotein B-containing lipoproteins. The Journal of biological chemistry. 2002; 277:1737780. [PubMed: 12006608]

17. Kamagate A, et al. FoxO1 mediates insulin-dependent regulation of hepatic VLDL production in mice. The Journal of clinical investigation. 2008; 118:2347-2364. [PubMed: 18497885]

18. Bartels ED, et al. Hepatic expression of microsomal triglyceride transfer protein and in vivo secretion of triglyceride-rich lipoproteins are increased in obese diabetic mice. Diabetes. 2002; 51:1233-1239. [PubMed: 11916950]

19. Sparks JD, et al. Acute suppression of apo B secretion by insulin occurs independently of MTP. Biochemical and biophysical research communications. 2011; 406:252-6. [PubMed: 21316344]

20. Lin MC, et al. Microsomal triglyceride transfer protein (MTP) regulation in HepG2 cells: insulin negatively regulates MTP gene expression. Journal of lipid research. 1995; 36:1073-81. [PubMed: 7658155]

21. Qiu W, et al. Overexpression of the endoplasmic reticulum 60 protein ER-60 downregulates apoB100 secretion by inducing its intracellular degradation via a nonproteasomal pathway: 
evidence for an ER-60-mediated and pCMB-sensitive intracellular degradative pathway. Biochemistry. 2004; 43:4819-31. [PubMed: 15096051]

22. Morand J, et al. Proteomic profiling of hepatic endoplasmic reticulum-associated proteins in an animal model of insulin resistance and metabolic dyslipidemia. The Journal of biological chemistry. 2005; 280:17626-17633. [PubMed: 15760893]

23. Pan M, et al. The late addition of core lipids to nascent apolipoprotein B100, resulting in the assembly and secretion of triglyceride-rich lipoproteins, is independent of both microsomal triglyceride transfer protein activity and new triglyceride synthesis. The Journal of biological chemistry. 2002; 277:4413-21. [PubMed: 11704664]

24. Brown AM, Gibbons GF. Insulin inhibits the maturation phase of VLDL assembly via a phosphoinositide 3-kinase-mediated event. Arteriosclerosis, thrombosis, and vascular biology. 2001; 21:1656-61.

25. Pollin T, et al. A null mutation in human APOC3 confers a favorable plasma lipid profile and apparent cardioprotection. Science. 2008; 322:1702-5. [PubMed: 19074352]

26. Ooi EMM, et al. Apolipoprotein C-III: understanding an emerging cardiovascular risk factor. Clinical science. 2008; 114:611-24. [PubMed: 18399797]

27. Sundaram M, et al. Functional analysis of the missense APOC3 mutation Ala23Thr associated with human hypotriglyceridemia. Journal of lipid research. 2010; 51:1524-34. [PubMed: 20097930]

28. Aalto-Setälä K, et al. Mechanism of hypertriglyceridemia in human apolipoprotein (apo) CIII transgenic mice. Diminished very low density lipoprotein fractional catabolic rate associated with increased apo CIII and reduced apo E on the particles. The Journal of clinical investigation. 1992; 90:1889-900. [PubMed: 1430212]

29. Caron S, et al. Transcriptional activation of apolipoprotein CIII expression by glucose may contribute to diabetic dyslipidemia. Arteriosclerosis, thrombosis, and vascular biology. 2011; 31:513-9.

30. Hernandez C, et al. Regulation of hepatic ApoC3 expression by PGC-1 $\beta$ mediates hypolipidemic effect of nicotinic acid. Cell metabolism. 2010; 12:411-9. [PubMed: 20889132]

31. Florez $\mathrm{H}$, et al. Increased apolipoprotein C-III levels associated with insulin resistance contribute to dyslipidemia in normoglycemic and diabetic subjects from a triethnic population. Atherosclerosis. 2006; 188:134-41. [PubMed: 16298371]

32. Sparks JD, et al. Insulin-mediated inhibition of apolipoprotein B secretion requires an intracellular trafficking event and phosphatidylinositol 3-kinase activation: studies with brefeldin A and wortmannin in primary cultures of rat hepatocytes. The Biochemical journal. 1996; 313(Pt 2):56774. [PubMed: 8573094]

33. Andreo U, et al. Insulin-Stimulated Degradation of Apolipoprotein B100: Roles of Class II Phosphatidylinositol-3-Kinase and Autophagy. PloS one. 2013; 8:e57590. [PubMed: 23516411]

34. Strong A, et al. Hepatic sortilin regulates both apolipoprotein B secretion and LDL catabolism. The Journal of clinical investigation. 2012; 122:2807-16. [PubMed: 22751103]

35. Chamberlain JM, et al. Insulin suppression of apolipoprotein B in McArdle RH7777 cells involves increased sortilin 1 interaction and lysosomal targeting. Biochem Biophys Res Commun. 2013; 430:66-71. [PubMed: 23159624]

36. Hermey G. The Vps10p-domain receptor family. Cellular and molecular life sciences. 2009; 66:2677-89. [PubMed: 19434368]

37. Dubé JB, et al. Sortilin: an unusual suspect in cholesterol metabolism: from GWAS identification to in vivo biochemical analyses, sortilin has been identified as a novel mediator of human lipoprotein metabolism. BioEssays. 2011; 33:430-7. [PubMed: 21462369]

38. Ai D, et al. Activation of ER stress and mTORC1 suppresses hepatic sortilin-1 levels in obese mice. The Journal of clinical investigation. 2012; 122:20-22.

39. Williams KJ, et al. Mechanisms by which lipoprotein lipase alters cellular metabolism of lipoprotein(a), low density lipoprotein, and nascent lipoproteins. Roles for low density lipoprotein receptors and heparan sulfate proteoglycans. The Journal of biological chemistry. 1992; 267:13284-92. [PubMed: 1320015]

40. Sundaram M, Yao Z. Recent progress in understanding protein and lipid factors affecting hepatic VLDL assembly and secretion. Nutrition \& metabolism. 2010; 7:35. [PubMed: 20423497] 
41. Mazzone T, et al. In vivo stimulation of low-density lipoprotein degradation by insulin. Diabetes. 1984; 33:333-338. [PubMed: 6323237]

42. Howard BV, et al. Integrated study of low density lipoprotein metabolism and very low density lipoprotein metabolism in non-insulin-dependent diabetes. Metabolism. 1987; 36:870-877. [PubMed: 3626866]

43. Kissebah AH, et al. Plasma low density lipoprotein transport kinetics in noninsulin-dependent diabetes mellitus. The Journal of clinical investigation. 1983; 71:655-67. [PubMed: 6338042]

44. Steinberg D. The LDL modification hypothesis of atherogenesis: an update. Journal of lipid research. 2009; 50(Suppl):S376-81. [PubMed: 19011257]

45. Vergès B. Lipid modification in type 2 diabetes: the role of LDL and HDL. Fundamental \& clinical pharmacology. 2009; 23:681-5. [PubMed: 19650852]

46. Adeli K, Lewis GF. Intestinal lipoprotein overproduction in insulin-resistant states. Current opinion in lipidology. 2008; 19:221-8. [PubMed: 18460911]

47. Brown MS, Goldstein JL. Regulation of the activity of the low density lipoprotein receptor in human fibroblasts. Cell. 1975; 6:307-316. [PubMed: 212203]

48. Chait A, et al. Regulatory role of insulin in the degradation of low density lipoprotein by cultured human skin fibroblasts. Biochimica et biophysica acta. 1978; 529:292-9. [PubMed: 207342]

49. Wade DP, et al. Regulation of low- density- lipoprotein- receptor mRNA by insulin in human hepatoma Hep G2 cells. European journal of biochemistry. 1989; 181:727-731. [PubMed: 2471639]

50. Costet P, et al. Hepatic PCSK9 expression is regulated by nutritional status via insulin and sterol regulatory element-binding protein 1c. The Journal of biological chemistry. 2006; 281:6211-6218. [PubMed: 16407292]

51. Niesen M, et al. Diabetes alters LDL receptor and PCSK9 expression in rat liver. Archives of biochemistry and biophysics. 2008; 470:111-115. [PubMed: 18054320]

52. Han S, et al. Hepatic insulin signaling regulates VLDL secretion and atherogenesis in mice. The Journal of clinical investigation. 2009; 119:1029-41. [PubMed: 19273907]

53. Biddinger SB, et al. Hepatic insulin resistance is sufficient to produce dyslipidemia and susceptibility to atherosclerosis. Cell metabolism. 2008; 7:125-134. [PubMed: 18249172]

54. Lundasen T, et al. Leptin induces the hepatic high density lipoprotein receptor scavenger receptor B type I (SR-BI) but not cholesterol 7alpha-hydroxylase (Cyp7a1) in leptin-deficient (ob/ob) mice. The Journal of biological chemistry. 2003; 278:43224-43228. [PubMed: 12917427]

55. Lambert G, et al. The PCSK9 decade. Journal of lipid research. 2012; 53:2515-24. [PubMed: 22811413]

56. Stein E, Mellis S. Effect of a monoclonal antibody to PCSK9 on LDL cholesterol. The New England journal of medicine. 2012; 366:1108-1118. [PubMed: 22435370]

57. Lillis AP, et al. LDL receptor-related protein 1: unique tissue-specific functions revealed by selective gene knockout studies. Physiological reviews. 2008; 88:887-918. [PubMed: 18626063]

58. Rohlmann A, et al. Inducible inactivation of hepatic LRP gene by cre-mediated recombination confirms role of LRP in clearance of chylomicron remnants. The Journal of clinical investigation. 1998; 101:689-95. [PubMed: 9449704]

59. Laatsch A, et al. Insulin stimulates hepatic low density lipoprotein receptor-related protein 1 (LRP1) to increase postprandial lipoprotein clearance. Atherosclerosis. 2009; 204:105-11. [PubMed: 18834984]

60. Ko KW, et al. The insulin-stimulated cell surface presentation of low density lipoprotein receptorrelated protein in 3T3-L1 adipocytes is sensitive to phosphatidylinositide 3-kinase inhibition. Biochemistry. 2001; 40:752-759. [PubMed: 11170392]

61. Jedrychowski MP, et al. Proteomic analysis of GLUT4 storage vesicles reveals LRP1 to be an important vesicle component and target of insulin signaling. The Journal of biological chemistry. 2010; 285:104-14. [PubMed: 19864425]

62. Bovenschen N, et al. Elevated plasma factor VIII in a mouse model of low-density lipoprotein receptor-related protein deficiency. Blood. 2003; 101:3933-9. [PubMed: 12522008] 
63. Basford JE, et al. Hepatic deficiency of low density lipoprotein receptor-related protein-1 reduces high density lipoprotein secretion and plasma levels in mice. The Journal of biological chemistry. 2011; 286:13079-87. [PubMed: 21343303]

64. Williams KJ, Chen K. Recent insights into factors affecting remnant lipoprotein uptake. Current opinion in lipidology. 2010; 21:218-28. [PubMed: 20463470]

65. Chen K, Williams KJ. Molecular mediators for raft-dependent endocytosis of syndecan-1, a highly conserved, multifunctional receptor. The Journal of biological chemistry. 2013

66. Stanford KI, et al. Syndecan-1 is the primary heparan sulfate proteoglycan mediating hepatic clearance of triglyceride-rich lipoproteins in mice. The Journal of clinical investigation. 2009; 119:3236-45. [PubMed: 19805913]

67. MacArthur JM, et al. Liver heparan sulfate proteoglycans mediate clearance of triglyceride-rich lipoproteins independently of LDL receptor family members. The Journal of clinical investigation. 2007; 117:153-64. [PubMed: 17200715]

68. Kjellén L, et al. Reduced sulfation of liver heparan sulfate in experimentally diabetic rats. Diabetes. 1983; 32:337-42. [PubMed: 6219905]

69. Bishop JR, et al. Insulin-dependent diabetes mellitus in mice does not alter liver heparan sulfate. The Journal of biological chemistry. 2010; 285:14658-62. [PubMed: 20236939]

70. Ebara T, et al. Delayed catabolism of apoB-48 lipoproteins due to decreased heparan sulfate proteoglycan production in diabetic mice. The Journal of clinical investigation. 2000; 105:18071818. [PubMed: 10862796]

71. Williams KJ, et al. Loss of heparan N-sulfotransferase in diabetic liver: role of angiotensin II. Diabetes. 2005; 54:1116-1122. [PubMed: 15793251]

72. Chen $\mathrm{K}$, et al. Type 2 diabetes in mice induces hepatic overexpression of sulfatase 2, a novel factor that suppresses uptake of remnant lipoproteins. Hepatology. 2010; 52:1957-67. [PubMed: 21049473]

73. Hassing HC, et al. Inhibition of hepatic sulfatase-2 in vivo: a novel strategy to correct diabetic dyslipidemia. Hepatology. 2012; 55:1746-53. [PubMed: 22234891]

74. Yen FT, et al. Molecular cloning of a lipolysis-stimulated remnant receptor expressed in the liver. The Journal of biological chemistry. 1999; 274:13390-8. [PubMed: 10224102]

75. Jun JY, et al. Spontaneously diabetic Ins2(+/Akita):apoE-deficient mice exhibit exaggerated hypercholesterolemia and atherosclerosis. American journal of physiology. Endocrinology and metabolism. 2011; 301:E145-54. [PubMed: 21447785]

76. Narvekar P, et al. Liver-specific loss of lipolysis-stimulated lipoprotein receptor triggers systemic hyperlipidemia in mice. Diabetes. 2009; 58:1040-9. [PubMed: 19188430]

77. Mendivil CO, et al. Metabolism of very-low-density lipoprotein and low-density lipoprotein containing apolipoprotein C-III and not other small apolipoproteins. Arteriosclerosis, thrombosis, and vascular biology. 2010; 30:239-45.

78. Musunuru K, et al. From noncoding variant to phenotype via SORT1 at the 1 p13 cholesterol locus. Nature. 2010; 466:714-9. [PubMed: 20686566]

79. Khamzina L, et al. Increased activation of the mammalian target of rapamycin pathway in liver and skeletal muscle of obese rats: possible involvement in obesity-linked insulin resistance. Endocrinology. 2005; 146:1473-81. [PubMed: 15604215]

80. Haas JT, et al. Hepatic insulin signaling is required for obesity-dependent expression of SREBP-1c mRNA but not for feeding-dependent expression. Cell metabolism. 2012; 15:873-84. [PubMed: 22682225]

81. Sørensen LP, et al. Basal and insulin mediated VLDL-triglyceride kinetics in type 2 diabetic men. Diabetes. 2011; 60:88-96. [PubMed: 20858686]

82. Lewis GF, et al. Effects of acute hyperinsulinemia on VLDL triglyceride and VLDL apoB production in normal weight and obese individuals. Diabetes. 1993; 42:833-842. [PubMed: 8495807]

83. Adiels M, et al. Overproduction of large VLDL particles is driven by increased liver fat content in man. Diabetologia. 2006; 49:755-65. [PubMed: 16463046]

84. Cummings $\mathrm{MH}$, et al. Increased hepatic secretion of very-low-density lipoprotein apolipoprotein B-100 in NIDDM. Diabetologia. 1995; 38:959-67. [PubMed: 7589883] 
85. Duvillard L, et al. Metabolic abnormalities of apolipoprotein B-containing lipoproteins in noninsulin-dependent diabetes: a stable isotope kinetic study. European journal of clinical investigation. 2000; 30:685-94. [PubMed: 10964160]

86. Kissebah $\mathrm{AH}$, et al. Integrated regulation of very low density lipoprotein triglyceride and apolipoprotein-B kinetics in non-insulin-dependent diabetes mellitus. Diabetes. 1982; 31:217-25. [PubMed: 7152129]

87. Brown M, Goldstein J. Selective versus total insulin resistance: a pathogenic paradox. Cell metabolism. 2008; 7:95-96. [PubMed: 18249166]

88. Stillemark-Billton $\mathrm{P}$, et al. Relation of the size and intracellular sorting of apoB to the formation of VLDL 1 and VLDL 2. Journal of lipid research. 2005; 46:104-14. [PubMed: 15520448]

89. Wu X, et al. Evidence for a lack of regulation of the assembly and secretion of apolipoprotein Bcontaining lipoprotein from HepG2 cells by cholesteryl ester. The Journal of biological chemistry. 1994; 269:12375-82. [PubMed: 8163541]

90. Adiels M, et al. Overproduction of VLDL1 driven by hyperglycemia is a dominant feature of diabetic dyslipidemia. Arteriosclerosis, thrombosis, and vascular biology. 2005; 25:1697-703.

91. Vergès B. New insight into the pathophysiology of lipid abnormalities in type 2 diabetes. Diabetes \& metabolism. 2005; 31:429-39. [PubMed: 16357786]

92. Mooradian A. Dyslipidemia in type 2 diabetes mellitus. Nature clinical practice endocrinology \& metabolism. 2009; 5:150-9.

93. Sugden MC, et al. Regulation of hepatic lipogenesis in starved and diabetic animals by thyroid hormone. Bioscience reports. 1981; 1:757-64. [PubMed: 6796152]

94. Taskinen MR, Nikkilä EA. Lipoprotein lipase activity of adipose tissue and skeletal muscle in insulin-deficient human diabetes. Relation to high-density and very-low-density lipoproteins and response to treatment. Diabetologia. 1979; 17:351-6. [PubMed: 231535]

95. Nikkilä EA, Kekki M. Plasma triglyceride transport kinetics in diabetes mellitus. Metabolism: clinical and experimental. 1973; 22:1-22. [PubMed: 4628966]

96. Pérez A, et al. Lipoprotein compositional abnormalities in type I diabetes: effect of improved glycaemic control. Diabetes research and clinical practice. 1997; 36:83-90. [PubMed: 9229192]

97. Lopes-Virella MF, et al. Plasma lipids and lipoproteins in young insulin-dependent diabetic patients: relationship with control. Diabetologia. 1981; 21:216-23. [PubMed: 7297793]

98. Sniderman AD, et al. Hypertriglyceridemic hyperapoB in type 2 diabetes. Diabetes care. 2002; 25:579-82. [PubMed: 11874951] 


\section{Highlights}

- In the liver, insulin antagonizes the ApoB secretion pathway at multiple sites

- Insulin promotes ApoB clearance via multiple hepatic receptor systems

- In diabetes, ApoB secretion is increased, and ApoB clearance is decreased

- Changes in ApoB metabolism may drive cardiovascular disease in the diabetic state 


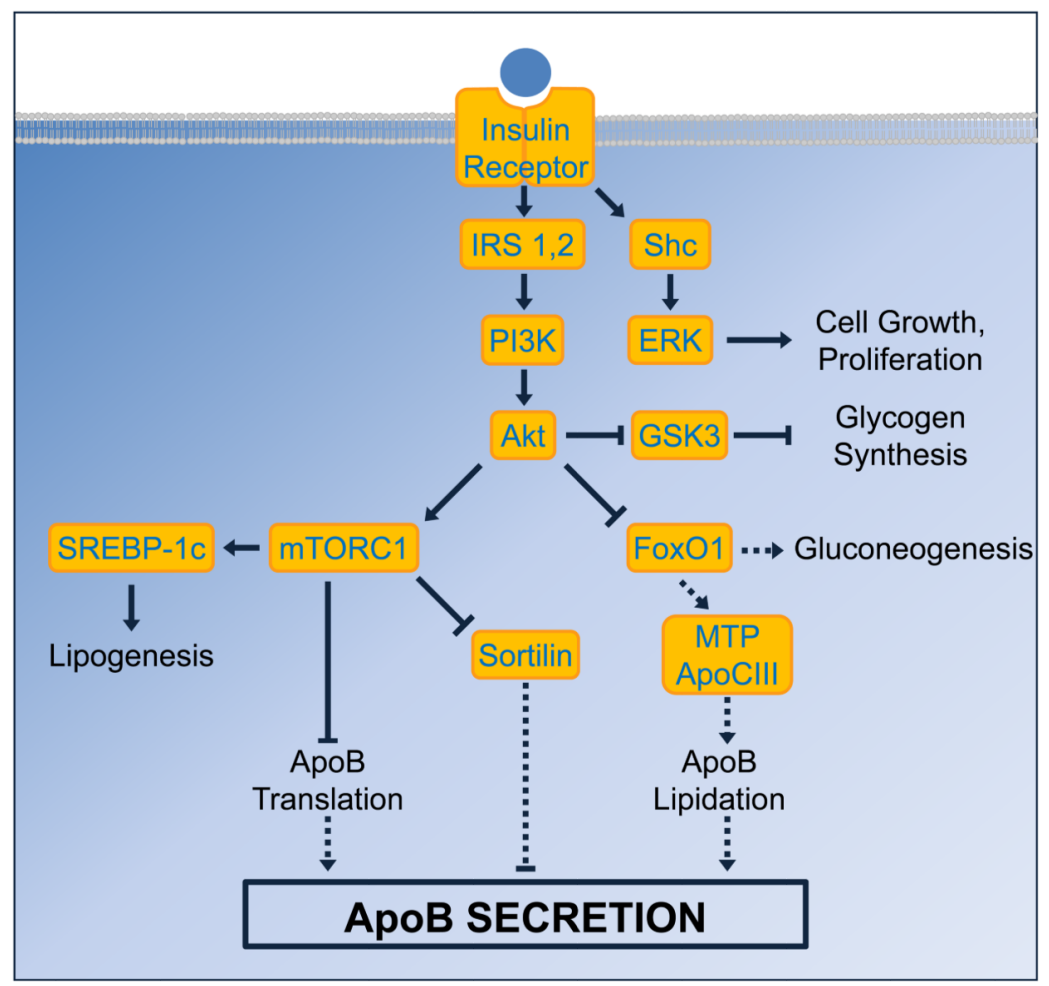

Figure 1. Insulin signaling cascade

Insulin binds to its receptor and recruits the insulin receptor substrate (IRS) proteins, leading to the activation of phosphatidylinositide 3-kinase (PI3K) and subsequently Akt. Akt serves as a major branch point in the signaling cascade: its inactivation of glycogen synthase kinase 3 (GSK3) de-represses and therefore stimulates glycogen synthesis, whereas its activation of mammalian target of rapamycin complex 1 (mTORC1) stimulates lipogenesis via the transcription factor SREBP-1c. Activation of mTORC1 also has dual effects on ApoB synthesis: it inhibits ApoB secretion by decreasing ApoB translation, but promotes ApoB secretion by inhibiting sortilin. The inhibition of FoxO1 by Akt represses expression of gluconeogenic genes and thus decreases gluconeogenesis; it also inhibits expression of MTP and ApoCIII, thereby inhibiting lipidation of ApoB and decreasing ApoB secretion. Finally, insulin can also activate extracellular signal-regulated kinase (ERK) via Shc to stimulate cell growth and proliferation. Solid arrows denote pathways that are active during insulin signaling; dashed arrows indicate pathways that are inactive during insulin signaling. 


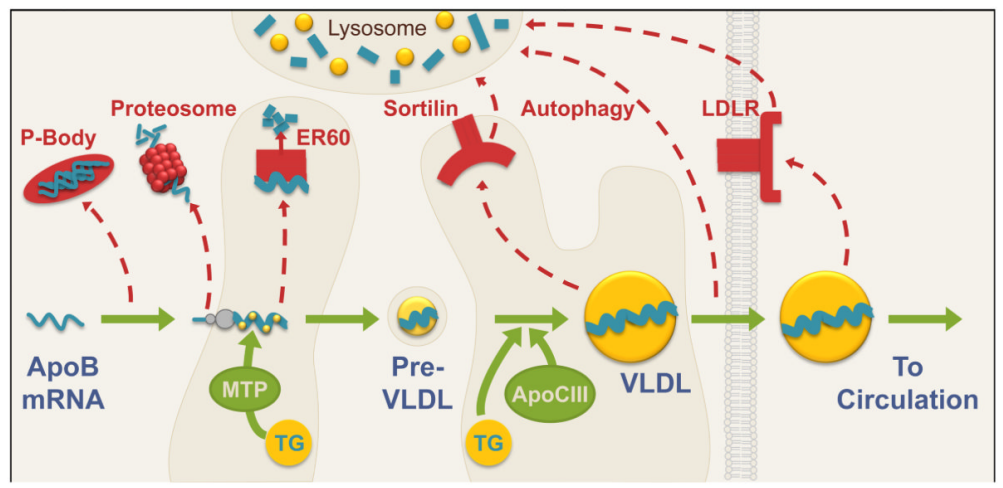

Figure 2. Synthesis of ApoB and secretion of VLDL

During translation, ApoB is lipidated with triglycerides (TG) by microsomal transfer protein (MTP) in the endoplasmic reticulum (ER) to form a lipid-poor pre-VLDL particle. The preVLDL particle is trafficked through the ER and Golgi compartments, where additional triglycerides are added in an MTP-independent manner promoted by ApoCIII, before secretion of the mature VLDL particle into the circulation. The synthesis and secretion of ApoB can be inhibited by multiple mechanisms, including proteasomal, ER60-mediated, autophagic and lysosomal degradation. Green arrows indicate ApoB-VLDL synthesis pathways; red dashed arrows indicate ApoB degradation pathways. 


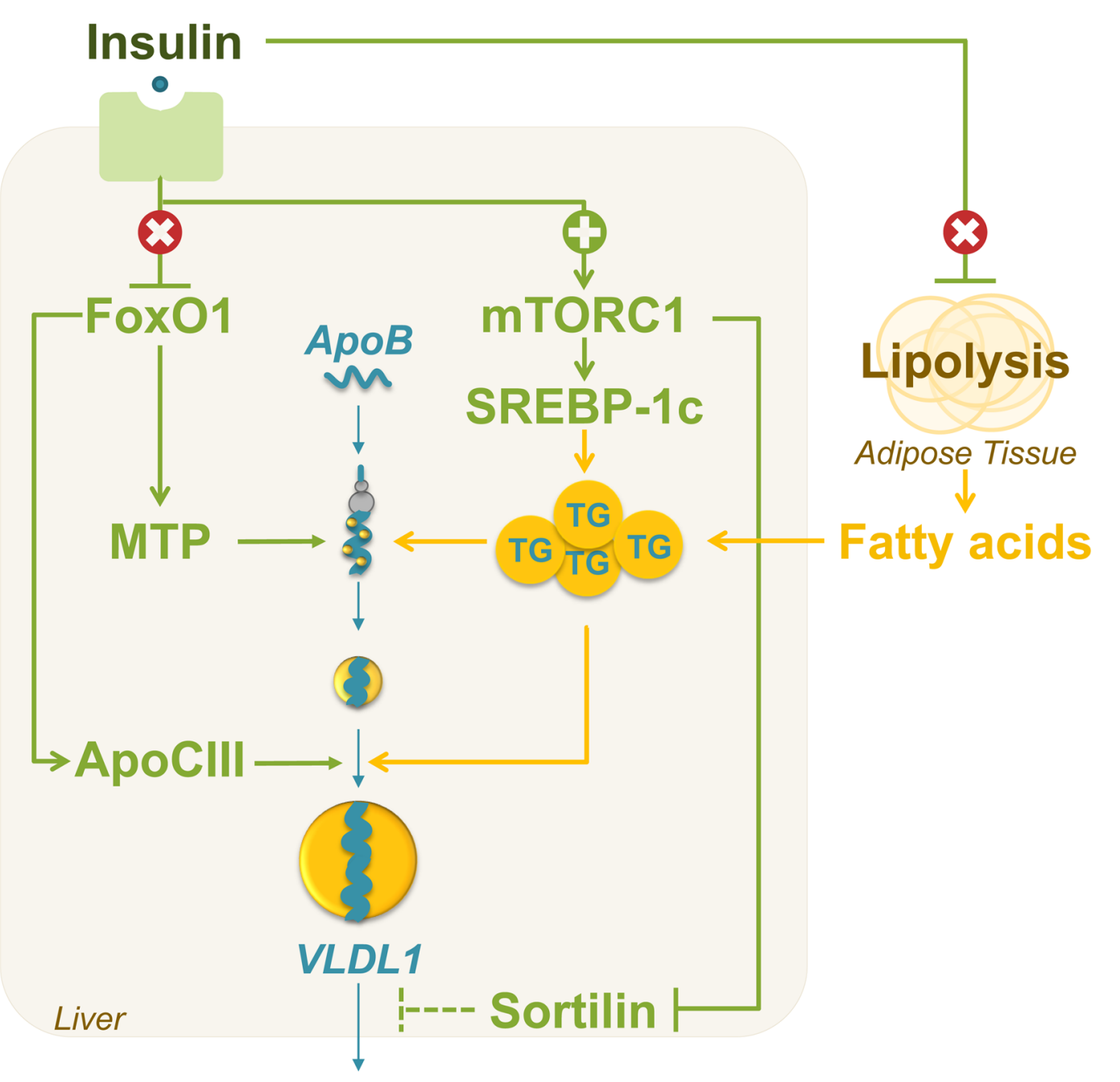

Figure 3. Type 2 diabetes drives VLDL secretion through multiple pathways

Type 2 diabetes is characterized by selective insulin resistance: insulin fails to suppress lipolysis in the adipose tissue and FoxO1 in the liver, but is still able to activate mTORC1. The dis-inhibition of FoxO1 leads to increased expression of MTP and ApoCIII, thereby promoting ApoB secretion. At the same time, the stimulation of mTORC1 in the liver leads to activation of SREBP-1c and increased lipogenesis, as well as suppression of sortilin. Because sortilin increases ApoB degradation, the suppression of sortilin further promotes ApoB secretion. Finally, the increase in lipogenesis coupled with the increased flux of fatty acids to the liver enlarges the pool of triglycerides available to lipidate ApoB, driving the formation of lipid-rich VLDL1 particles. The overall result is an increase in the secretion of ApoB in the form of VLDL1 particles in T2D. 